\title{
SPECTRUM AND MORPHOLOGY OF THE TWO BRIGHTEST MILAGRO SOURCES IN THE CYGNUS REGION: MGRO J2019+37 AND MGRO J2031+41
}

\author{
A. A. Abdo ${ }^{1,15}$, U. Abeysekara ${ }^{1}$, B. T. Allen ${ }^{2,16}$, T. Aune ${ }^{3}$, D. Berley ${ }^{4}$, E. Bonamente ${ }^{5}$, G. E. Christopher ${ }^{6,17}$, \\ T. DeYoung ${ }^{7}$, B. L. Dingus ${ }^{8}$, R. W. Ellsworth ${ }^{9}$, J. G. Galbraith-Frew ${ }^{5}$, M. M. Gonzalez ${ }^{10}$, J. A. Goodman $^{4}$, \\ C. M. Hoffman ${ }^{8}$, P. H. Hüntemeyer ${ }^{5}$, C. M. Hui ${ }^{5}$, B. E. Kolterman ${ }^{6}$, J. T. Linnemann ${ }^{1}$, J. E. McEnery ${ }^{11}$, A. I. Mincer ${ }^{6}$, \\ T. Morgan ${ }^{12}$, P. Nemethy ${ }^{6}$, J. Pretz ${ }^{8}$, J. M. Ryan ${ }^{12}$, P. M. Saz Parkinson ${ }^{3}$, A. Shoup ${ }^{13}$, G. Sinnis ${ }^{8}$, A. J. Smith ${ }^{4}$, \\ V. Vasileiou ${ }^{4,14}$, G. P. Walker ${ }^{8,18}$, D. A. Williams ${ }^{3}$, and G. B. Yodh $^{2}$ \\ ${ }^{1}$ Department of Physics and Astronomy, Michigan State University, 3245 BioMedical Physical Sciences Building, East Lansing, MI 48824, USA \\ ${ }^{2}$ Department of Physics and Astronomy, University of California, Irvine, CA 92697, USA \\ ${ }^{3}$ Santa Cruz Institute for Particle Physics, University of California, 1156 High Street, Santa Cruz, CA 95064, USA \\ ${ }^{4}$ Department of Physics, University of Maryland, College Park, MD 20742, USA \\ ${ }^{5}$ Department of Physics, Michigan Technological University, Houghton, MI 49931, USA; ebonamen@ mtu.edu, petra@mtu.edu \\ ${ }^{6}$ Department of Physics, New York University, 4 Washington Place, New York, NY 10003, USA \\ ${ }^{7}$ Department of Physics, Pennsylvania State University, University Park, PA 16802, USA \\ ${ }^{8}$ Group P-23, Los Alamos National Laboratory, P.O. Box 1663, Los Alamos, NM 87545, USA \\ ${ }^{9}$ Department of Physics and Astronomy, George Mason University, 4400 University Drive, Fairfax, VA 22030, USA \\ ${ }^{10}$ Instituto de Astronomía, Universidad Nacional Autónoma de México, 74D.F., México 04510, Mexico \\ ${ }^{11}$ NASA Goddard Space Flight Center, Greenbelt, MD 20771, USA \\ ${ }^{12}$ Department of Physics, University of New Hampshire, Morse Hall, Durham, NH 03824, USA \\ ${ }^{13}$ Department of Physics, The Ohio State University, Lima, OH 45804, USA \\ ${ }^{14}$ CRESST NASA/Goddard Space Flight Center, MD 20771 and University of Maryland, Baltimore County, MD 21250, USA \\ Received 2012 February 3; accepted 2012 May 10; published 2012 June 25
}

\begin{abstract}
The Cygnus region is a very bright and complex portion of the TeV sky, host to unidentified sources and a diffuse excess with respect to conventional cosmic-ray propagation models. Two of the brightest $\mathrm{TeV}$ sources, MGRO J2019+37 and MGRO J2031+41, are analyzed using Milagro data with a new technique, and their emission is tested under two different spectral assumptions: a power law and a power law with an exponential cutoff. The new analysis technique is based on an energy estimator that uses the fraction of photomultiplier tubes in the observatory that detect the extensive air shower. The photon spectrum is measured in the range $1-100 \mathrm{TeV}$ using the last three years of Milagro data (2005-2008), with the detector in its final configuration. An $F$-test indicates that MGRO J2019+37 is better fit by a power law with an exponential cutoff than by a simple power law. The best-fitting parameters for the power law with exponential cutoff model are a normalization at $10 \mathrm{TeV}$ of $7_{-2}^{+5} \times 10^{-10} \mathrm{~s}^{-1} \mathrm{~m}^{-2} \mathrm{TeV}^{-1}$, a spectral index of $2.0_{-1.0}^{+0.5}$, and a cutoff energy of $29_{-16}^{+50} \mathrm{TeV}$. MGRO $\mathrm{J} 2031+41$ shows no evidence of a cutoff. The best-fitting parameters for a power law are a normalization of $2.1_{-0.6}^{+0.6} \times$ $10^{-10} \mathrm{~s}^{-1} \mathrm{~m}^{-2} \mathrm{TeV}^{-1}$ and a spectral index of $3.22_{-0.18}^{+0.23}$. The overall flux is subject to a $\sim 30 \%$ systematic uncertainty. The systematic uncertainty on the power-law indices is $\sim 0.1$. Both uncertainties have been verified with cosmic-ray data. A comparison with previous results from TeV J2032+4130, MGRO J2031+41, and MGRO J2019+37 is also presented.
\end{abstract}

Key words: acceleration of particles - astroparticle physics - gamma rays: general - open clusters and associations: individual (Cyg OB1, Cyg OB2) - pulsars: general

Online-only material: color figures

\section{INTRODUCTION}

The Cygnus region is a part of our Galaxy of active massive star formation and destruction, and has been studied over a broad range of wavelengths, including radio, $\mathrm{X}$-ray, $\mathrm{GeV}$, and $\mathrm{TeV}$ gamma ray, as well as in cosmic rays. From $\mathrm{GeV}$ up to multi-TeV energies, the Cygnus region is the brightest diffuse gamma-ray source in the northern hemisphere (Hunter et al. 1997).

\footnotetext{
${ }^{15}$ Current address: Space Science Division, Naval Research Laboratory, Washington, DC 20375, USA.

${ }^{16}$ Current address: Harvard-Smithsonian Center for Astrophysics, Cambridge, MA 02138, USA.

${ }^{17}$ Current address: Department of Physics, Brown University, Providence, RI 02912, USA.

${ }^{18}$ Current address: National Security Technologies, Las Vegas, NV 89102, USA.
}

One of the challenges in analyzing the Cygnus region at $\mathrm{TeV}$ energies is the proper separation of the gamma-ray flux that is attributed to the point or extended sources in the region or to the diffuse emission. Previous Milagro analyses computed the diffuse emission from the region using two alternative methods to isolate the contribution from the resolved sources (Abdo et al. 2007a, 2008), and found that at $\mathrm{TeV}$ energies the flux is still in excess with respect to the predicted flux from the GALPROP model (Strong \& Moskalenko 1998; Moskalenko et al. 1998, 2000). Milagro also published the discovery of two unidentified $\mathrm{TeV}$ sources in the region, MGRO J2019+37 and MGRO J2031+41 (Abdo et al. 2007b, 2009b). The location of MGRO J2019+37 was found to be consistent with two EGRET sources, while the best-fit position for MGRO J2031+41 was near two EGRET sources and the HEGRA unidentified source $\mathrm{TeV}$ J2032+4130. In a correlation study connecting the $\mathrm{TeV}$ 
sources discovered by Milagro with sources detected above $10 \sigma$ (the so-called Bright Source List, BSL) by the Fermi Large Area Telescope (LAT), the two aforementioned brightest Milagro sources in the region were associated with GeV pulsars (Abdo et al. 2009a, 2009b). MGRO J2019+37 and MGRO J2031+41 are currently associated with two and one pulsars identified by the Fermi LAT, respectively (The Fermi LAT Collaboration $2012 b$ ). Milagro also detected two candidate sources in the Cygnus region $\left(l \in\left[65^{\circ}, 85^{\circ}\right]\right.$ and $b \in\left[-2^{\circ},+2^{\circ}\right]$; Abdo et al. 2009b).

Recently, several collaborations have presented new surveys of the Cygnus region, confirming the complexity of the region and showing the highly structured diffuse emission. The TeV emission from the position of MGRO J2019+37 has been confirmed by the VERITAS experiment (Aliu et al. 2011). The VERITAS spatial counts map shows a clear structure associated with MGRO J2019+37, a region of extended emission which seems to be produced by previously unresolved sources. At lower energies, the Fermi LAT Collaboration (Ackermann et al. 2011) has also recently published new results on the diffuse emission from the Cygnus region. An extended source, the socalled Cocoon, overlapping with MGRO J2031+41, has been found and its emission has been explained by freshly accelerated cosmic rays, trapped in a shell of photon-dominated emission formed by stellar winds and supernovae, as shown by midinfrared observations. The spectrum from this region is hard, extending up to $100 \mathrm{GeV}$ with no evidence of softening, and could be explained as the result of pulsar-accelerated particles or as an active super-bubble. The average diffuse emission from the region has also been analyzed at $\mathrm{MeV}$ to $\mathrm{GeV}$ energies (The Fermi LAT Collaboration 2012a). Despite the very rich source population, this emission is similar to that of the local interstellar space, and there is no evidence that it is necessary to include an extra contribution in the model, resembling the diffuse excess previously measured by Milagro (Abdo et al. 2007a, 2008). Most recently, the ARGO-YBJ collaboration presented the results of a survey of the Cygnus region in the energy range of $600 \mathrm{GeV}$ to $7 \mathrm{TeV}$. MGRO J2031+41 is observed with a significance of $6.4 \sigma$ and a flux consistent with previous Milagro results, but there is no significant detection of MGRO J2019+37 (Bartoli et al. 2012).

Here, we present a new analysis of the last three years of data collected with the Milagro experiment (2005-2008). An improved gamma-hadron separation and a newly developed technique are exploited to reconstruct the energy spectra of gamma rays from the sources in the Cygnus region. The best fits for the spectra of the two brightest Milagro sources are presented: MGRO J2019+37, a source observed in the complete Milagro data set collected over eight years of operation with a pre-trials significance in excess of $12 \sigma$ between 1 and $100 \mathrm{TeV}$, and MGRO J2031+41, a source observed in the complete data set with a pre-trials significance in excess of $7 \sigma$ (Abdo et al. 2009b). We compare our spectra with results from the HEGRA, MAGIC, Whipple, and ARGO-YBJ experiments (Aharonian et al. 2005; Albert et al. 2008; Lang et al. 2004; Bartoli et al. 2012).

\section{ANALYSIS TECHNIQUE}

The Milagro detector was located in the Jemez Mountains in New Mexico, at an altitude of $2630 \mathrm{~m}$ a.s.1. It was operated from 2001 to 2008, and, in its final configuration, consisted of two components: (1) a central pond $\left(60 \times 80 \mathrm{~m}^{2}, 8 \mathrm{~m}\right.$ deep $)$ with two photomultiplier tube (PMT) layers, a shallow air shower layer consisting of 450 PMTs, and a deep muon layer consisting of 273 PMTs and (2) an array of 175 single-PMT tank detectors surrounding the pond covering an area of 40,000 $\mathrm{m}^{2}$ (Atkins et al. 2003).

A detailed description of the analysis method and the parameters used here can be found in the Milagro paper on the spectral measurement of the Crab Nebula (Abdo et al. 2012). The method has been confirmed to be applicable for cosmic- and gamma-ray energies between 1 and $100 \mathrm{TeV}$. Using cosmic-ray data, systematic uncertainties of this method are estimated to be $\sim 30 \%$ on the overall flux and $\sim 0.1$ on the spectral index (see Section 4 of Abdo et al. 2012 for more details).

The new element in Abdo et al. (2012) and this paper, with respect to the previous approach (Abdo et al. 2007b, 2009b), is the introduction of an estimator, $\mathcal{F}$, for the energy of the primary particle initiating the extensive air shower, based on the number of PMTs hit for each event. The $\mathcal{F}$ parameter is defined as the sum of two fractions:

$$
\mathcal{F}=\frac{N_{\text {A.S. }}}{N_{\text {A.S. }}^{\text {live }}}+\frac{N_{\text {T.A. }}}{N_{\text {T.A. }}^{\text {live }}},
$$

where $N_{\text {A.S. }}$ and $N_{\mathrm{s}}$ are the number of PMTs detecting the event, while $N_{\text {A.S. }}^{\text {live }}$ and $N_{\text {T.A. }}^{\text {live }}$ are the number of functional PMTs in the air shower layer and in the tank array, respectively. Because the typical energy resolution of Milagro in each $\mathcal{F}$ bin is quite broad $(50 \%-100 \%)$, the energy distributions between $\mathcal{F}$ bins overlap, and the median energy associated with a given $\mathcal{F}$ value is dependent on the spectral assumption for the observed source, the fit is performed in the measured $\mathcal{F}$ space rather than in the energy space.

The reconstructed Milagro data contain information about the direction and $\mathcal{F}$ value of air shower events. According to their $\mathcal{F}$ value, events are sorted into nine bins $(0.2 \leqslant \mathcal{F} \leqslant 2)$, resulting in a total of nine signal skymaps, binned in $0.1 \times 0.1$ pixels. For each $\mathcal{F}$ bin, background maps are calculated using the Direct Integration method with the typical $2 \mathrm{hr}$ integration duration (Atkins et al. 2003; Abdo et al. 2012). The two brightest regions of interest in the sky, a $2^{\circ} \times 2^{\circ}$ area around the position of the Crab Nebula and a band around the Galactic plane $(-2.5<b<2.5)$, are excluded when calculating the background (Abdo et al. 2012). Rather than discriminating between gamma-ray and cosmic-ray initiated air showers, a weight is applied to all measured events, in both signal and background maps, where gamma-ray-like events are assigned a higher weight than cosmic-ray-like events (Abdo et al. 2012). The gamma-ray excess with respect to the estimated background is calculated as the difference between signal weights and background weights, and is filled into the so-called excess map. Based on these excess maps, we compute the $\mathcal{F}$ distributions used in the energy fit.

Excess maps are smoothed according to the detector angular resolution (or point-spread function, PSF), which is a function of $\mathcal{F}$ and varies between 0.3 and 0.7 . The measured $\mathcal{F}$ distribution for the target source in a $0.1 \times 0.1 \mathrm{bin}$, as a result, is the average excess coming from a PSF-wide region around the nominal source position. Since the background is measured with the direct integration method removing the Galactic plane ( $b>2.5$ and $b<-2.5$ ), the measured excess from sources in the Galactic plane includes any other diffuse or extended emission possibly present in the vicinity of the source. The Galactic diffuse background was estimated to contribute up to $15 \%$ of the flux at $35 \mathrm{TeV}$ for the weakest Galactic BSL source (Abdo et al. 2009b). 
Table 1

Source Positions

\begin{tabular}{|c|c|c|c|c|}
\hline Survey & $\begin{array}{l}\text { R.A. } \\
(\text { deg })\end{array}$ & $\begin{array}{l}\text { Decl. } \\
(\mathrm{deg})\end{array}$ & $\begin{array}{c}1 \sigma \text { Error Ellipse (Semi) } \\
(\mathrm{deg})\end{array}$ & $\begin{array}{l}\text { Width }(\sigma) \\
\quad(\operatorname{deg})\end{array}$ \\
\hline \multicolumn{5}{|l|}{ MGRO J2019+37 } \\
\hline Two-dimensional Gaussian ${ }^{\mathrm{a}}$ & 304.63 & 36.88 & $0.11,0.08$ & 0.70 \\
\hline Fermi position (2FGL J2021.0+3651) ${ }^{\mathrm{c}}$ & 305.2702 & 36.8634 & $0.0081,0.0078$ & $\ldots$ \\
\hline Milagro $2007^{\mathrm{d}}$ & 304.98 & 36.66 & $0.20,0.13$ & $1.1^{\mathrm{d}}$ \\
\hline \multicolumn{5}{|l|}{ MGRO J2031+41 } \\
\hline Two-dimensional Gaussian ${ }^{\mathrm{a}, \mathrm{b}}$ & 307.18 & 41.31 & $0.42,0.26$ & 1.8 \\
\hline Fermi position (2FGL J2032.2+4126) ${ }^{\mathrm{c}}$ & 308.0622 & 41.4369 & $0.0142,0.0133$ & \\
\hline Milagro $2007^{\mathrm{d}}$ & 307.98 & 41.51 & $0.52,0.33$ & $3.0^{\mathrm{d}}$ \\
\hline
\end{tabular}

Notes. Positions of MGRO J2019+37 (top) and MGRO J2031+41 (bottom) for the two-dimensional Gaussian fit, Fermi position of associated sources and previous Milagro survey.

${ }^{a}$ The table lists statistical errors only. The systematic pointing error is $<0.3$.

${ }^{\mathrm{b}}$ Studies with subsets of data result in an additional systematic uncertainty of $<0.5$ in the case of MGRO J2031+41.

${ }^{c}$ Abdo et al. (2009b).

${ }^{\mathrm{d}}$ The number reported is the diameter of a top-hat function that best fits the angular extent of the source (Abdo et al. 2007b).

We test the emission from the two sources for two different spectral hypotheses: a power law, defined as

$$
\frac{d N}{d E}=N_{10 \mathrm{TeV}}\left(\frac{E}{10 \mathrm{TeV}}\right)^{-\alpha}
$$

where $N_{10 \mathrm{TeV}}$ is the normalization scale set at $10 \mathrm{TeV}$ and $\alpha$ is the spectral index, and a power law with an exponential cutoff, defined as

$$
\frac{d N}{d E}=N_{10 \mathrm{TeV}}\left(\frac{E}{10 \mathrm{TeV}}\right)^{-\alpha} \exp \left(-\frac{E}{E_{c}}\right)
$$

where $E_{c}$ is the cutoff energy.

The fit to the data is performed comparing the measured excess to simulations. First, a set of simulated data is generated varying sensitive parameters (i.e., $N_{10 \mathrm{TeV}}, \alpha$, and $E_{c}$ ). The best spectral parameters and the corresponding fit probability are then found using a $\chi^{2}$ minimization, comparing the measured and the simulated $\mathcal{F}$ distributions. Uncertainties on fit parameters are computed using $1 \sigma$ contours of $\chi^{2}$ histograms (as discussed in Sections 3.1 and 3.2). The uncertainty is defined as the distance between the best-fit value and the lower and upper edges of the $1 \sigma$ contour.

\section{RESULTS AND DISCUSSION}

The results presented here are obtained from the analysis of the Milagro data taken with the detector in its final configuration. The start date is 2005 October 22 20:39:16 GMT and the stop date is 2008 April 15 00:02:53 GMT, corresponding to a total of 906 days of observation, resulting in 832 integrated days after data quality cuts.

The positions of MGRO J2019+37 and MGRO J2031+41 are obtained fitting Milagro excess maps with a two-dimensional Gaussian function with equal widths in right ascension and declination (where the right ascension is corrected to get the true spherical distance). The fit is performed in a square region of $\pm 3^{\circ}$ around the excess. Table 1 shows the positions used in this paper and a comparison to other surveys. Table 2 lists the total gamma-ray event excess in each $\mathcal{F}$ bin for the two sources.

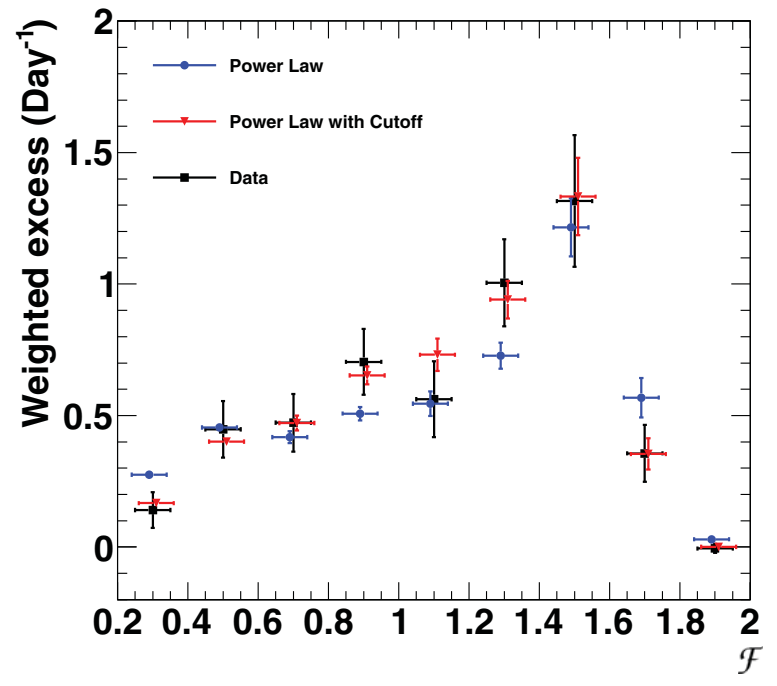

Figure 1. MGRO J2019+37: distributions of the parameter used to estimate the photon spectrum, $\mathcal{F}$. The spectrum fit is performed in the $\mathcal{F}$ space. Black squares represent the data. Red triangles and blue circles represent the simulated distributions for the best fit assuming a power law with a cutoff and a simple power law, respectively. The unit on the $y$-axis is the weighted excess per day, the unit on the $x$-axis is the $\mathcal{F}$ value. The error bars are the statistical uncertainties. (A color version of this figure is available in the online journal.)

\subsection{MGRO J2019+37 Spectrum}

In Figure 1, the $\mathcal{F}$ distribution of data is shown with the two simulated $\mathcal{F}$ distributions for the best-fitting power law and power law with exponential cutoff models. These distributions show the weighted excess coming from the source position after background subtraction. The excess from MGRO J2019+37 is compared to the excess measured from the entire Cygnus region $\left(65^{\circ}<l<85^{\circ}\right.$ and $\left.-2^{\circ}<b<2^{\circ}\right)$. We find that the emission from MGRO J2019+37 accounts for $\sim 12 \%$ of the excess from the entire region.

Figures 2 and 3 show the $1 \sigma$ and $2 \sigma$ contours from the $\chi^{2}$ fit for the two tested hypotheses. $1 \sigma$ contours are used to compute uncertainty on the parameters as described in Section 2.

The best-fit spectra for the two tested hypotheses (Equations (2) and (3)) are shown in Figure 4. The best-fit spectral parameters are summarized in Table 3. 


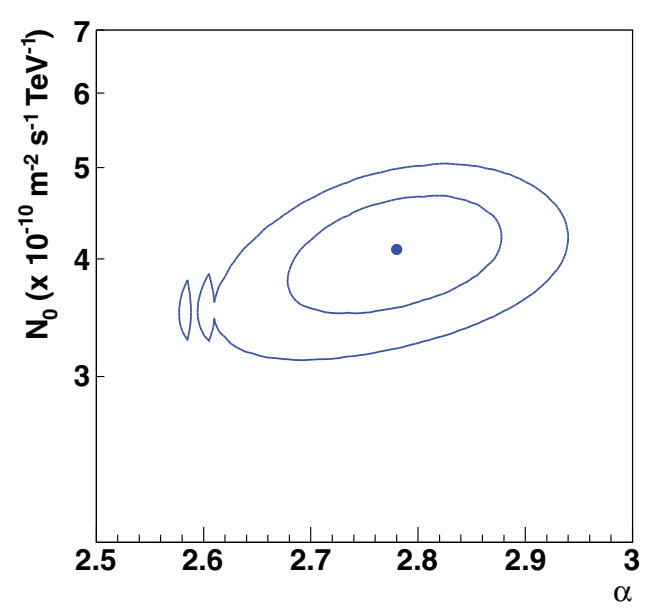

Figure 2. MGRO J2019+37: $1 \sigma$ and $2 \sigma$ CL contours for the power-law fit. The point indicates the best-fit result (minimum $\chi^{2}$ ). The $\chi^{2}$ increments for the $1 \sigma$ and $2 \sigma$ contours are 2.30 and 6.18 , respectively.

(A color version of this figure is available in the online journal.)

With the power-law hypothesis, the best-fit parameters for the normalization and the spectral index are $N_{10 \mathrm{TeV}}=\left(4.1_{-0.6}^{+0.6}\right) \times$ $10^{-10} \mathrm{~s}^{-1} \mathrm{~m}^{-2} \mathrm{TeV}^{-1}$ and $\alpha=2.78_{-0.10}^{+0.10}$, respectively. The $\chi^{2}$ is 16.12 for 7 degrees of freedom (dof), which gives a $\chi^{2}$ fit probability of 0.024 .

In the case of the power law with cutoff hypothesis, the best-fit parameters are $N_{10 \mathrm{TeV}}=7_{-2}^{+5} \times 10^{-10} \mathrm{~s}^{-1} \mathrm{~m}^{-2} \mathrm{TeV}^{-1}$, $\alpha=2.0_{-1.0}^{+0.5}$, and $E_{c}=29_{-16}^{+50} \mathrm{TeV}$. The $\chi^{2}$ is 1.924 (6 dof), which gives a $\chi^{2}$ fit probability of 0.93 .

The $\chi^{2}$ fit probabilities suggest that the power law with a cutoff model $\left(\chi^{2}\right.$ probability $\left.=93 \%\right)$ fits the data better than a simple power law $\left(\chi^{2}\right.$ probability $\left.=2.4 \%\right)$. An $F$-test (Bevington \& Robinson 2003; Bates \& Watts 2007) for the drop in $\chi^{2}$ when adding the cutoff parameter to the fit gives a probability of $6 \times 10^{-4}$ of having seen a larger change, if the simpler power law without exponential cutoff were the correct model. To test the robustness of the result, since the highest $\mathcal{F}$ bins may be subject to larger systematic uncertainties, we repeat the spectral fit excluding the last, and the last two bins of the distribution. The extracted parameter values do not change significantly, and despite the inclusion of fewer data, the simple power law remains disfavored. The $F$-test for the drop in $\chi^{2}$ when adding the cutoff parameter to the spectral fit in only 8 (7)

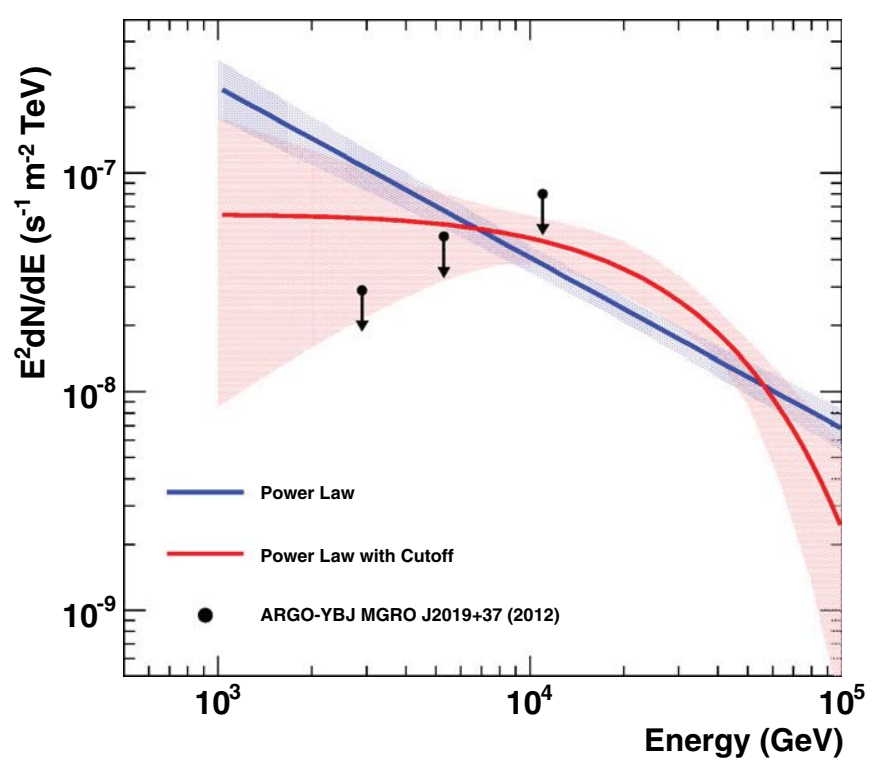

Figure 4. MGRO J2019+37: energy spectra. The best fit is obtained for a power law with cutoff model (in red). The power-law model is also shown (in blue). The shadowed area represents the $1 \sigma$ band, obtained by varying the parameters within the $1 \sigma$ contour. ARGO-YBJ 90\% CL upper limits for MGRO J2019+37 are shown in black (Bartoli et al. 2012).

(A color version of this figure is available in the online journal.)

$F$ bins gives a probability of $4 \times 10^{-3}\left(6 \times 10^{-2}\right)$ of having seen a larger change, if the simpler power law without exponential cutoff were the correct model.

Previous Milagro analyses quoted the flux of MGRO J2019+37 at 20 and $35 \mathrm{TeV}$, respectively (Abdo et al. 2007b, 2009b). Those values, using a different analysis technique, are in agreement with the results presented here. An independent analysis (Allen 2007), which used a different energy estimator for the particle initiating the air shower and a different parameter to distinguish between gamma and cosmic rays, is also consistent with our results. ARGO-YBJ (Bartoli et al. 2012) has not observed any significant emission from MGRO J2019+37 in the range $600 \mathrm{GeV}$ to $7 \mathrm{TeV}$. The explanations put forward in (Bartoli et al. 2012) are an insufficient exposure above 5 $\mathrm{TeV}$ or a possible time variability of MGRO J2019+37. Due to the extension of MGRO J2019+37, the latter explanation is not without problems. The $90 \%$ confidence level (CL) upper limit from ARGO-YBJ is consistent with our best-fitting model, a power law with an exponential cutoff (see Figure 4). On the
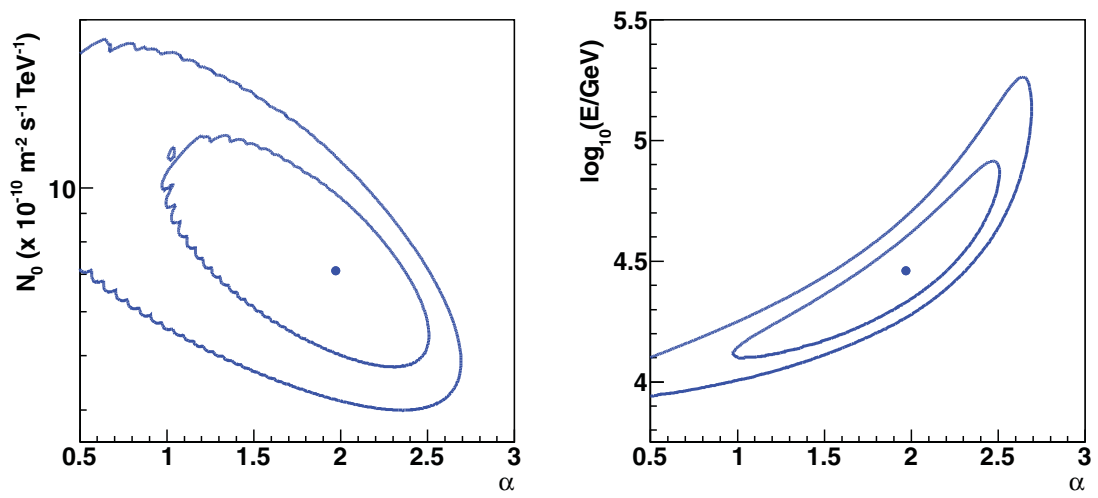

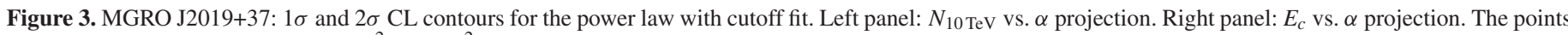
indicate the best-fit result (minimum $\chi^{2}$ ). The $\chi^{2}$ increments for the $1 \sigma$ and $2 \sigma$ contours are 3.53 and 8.02, respectively.

(A color version of this figure is available in the online journal.) 
Table 2

Weighted Gamma-ray Event Excess

\begin{tabular}{|c|c|c|c|c|c|c|c|c|c|}
\hline $\mathcal{F}$ & $0.2-0.4$ & $0.4-0.6$ & $0.6-0.8$ & $0.8-1.0$ & $1.0-1.2$ & $1.2-1.4$ & $1.4-1.6$ & $1.6-1.8$ & $1.8-2.0$ \\
\hline MGRO J2019+37 & $117_{-57}^{+57}$ & $372_{-90}^{+90}$ & $\begin{array}{r}393_{-91}^{+91} \\
\end{array}$ & $586_{-104}^{+104}$ & $468_{-120}^{+120}$ & $836_{-137}^{+137}$ & $1095_{-208}^{+208}$ & $296_{-90}^{+90}$ & $\begin{array}{l}-4_{-14}^{+14} \\
\end{array}$ \\
\hline MGRO J2031+41 & $218_{-57}^{+57}$ & $216_{-90}^{+90}$ & $284_{-92}^{+92}$ & $277_{-106}^{+106}$ & $291_{-122}^{+122}$ & $162_{-143}^{+143}$ & $2_{-214}^{+214}$ & $100_{-86}^{+86}$ & $-4_{-11}^{+11}$ \\
\hline
\end{tabular}

Notes. Total weighted gamma-ray event excess in each $\mathcal{F}$ bin in 832 integrated days of data.

Table 3

Fit Results

\begin{tabular}{lcccc}
\hline \hline Source & $N_{10 \mathrm{TeV}}\left(10^{-10} \mathrm{~s}^{-1} \mathrm{~m}^{-2} \mathrm{TeV}^{-1}\right)$ & $\alpha$ & $E_{c}(\mathrm{TeV})$ & $\chi^{2} / \mathrm{dof}$ \\
\hline MGRO J2019+37 & $4.1_{-0.6}^{+0.6}$ & $2.78_{-0.10}^{+0.10}$ & $\infty$ & $16.12 / 7$ \\
MGRO J2019+37 & $7_{-2}^{+5}$ & $2.0_{-1.0}^{+0.5}$ & $29_{-16}^{+50}$ & $1.924 / 6$ \\
\hline MGRO J2031+41 & $2.1_{-0.6}^{+0.6}$ & $3.22_{-0.18}^{+0.23}$ & $\infty$ & 0.93 \\
MGRO J2031+41 & $5_{-3}^{+157}$ & $2.7_{-3.3}^{+0.7}$ & $21_{-18}^{+\infty}$ & $5.059 / 7$ \\
\hline
\end{tabular}

Notes. Best-fit results for MGRO J2019+37 and MGRO J2031+41 using both the power law and the power law with cutoff hypotheses. The table lists statistical errors only, the systematic flux error is $\sim 30 \%$ and the systematic error on the spectral index is $\sim 0.1$.

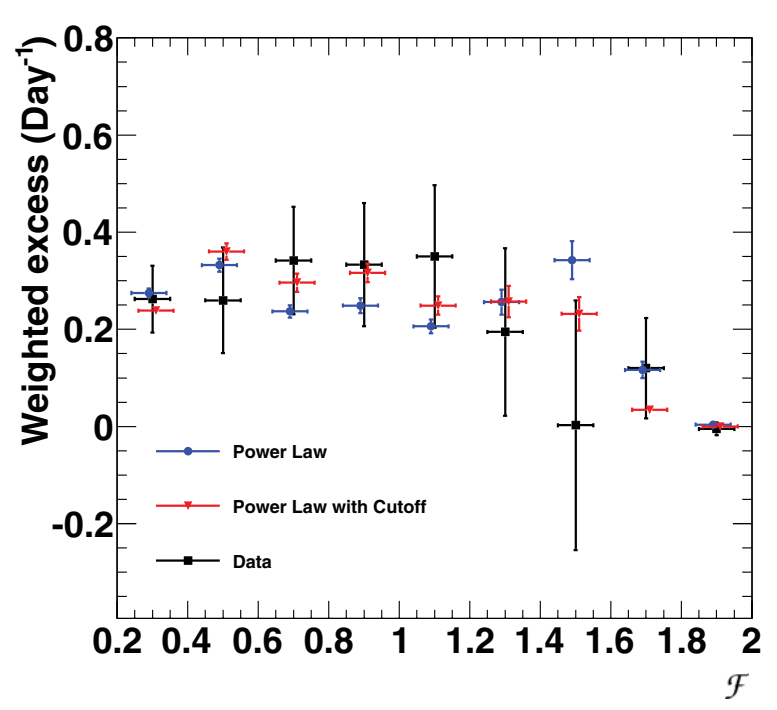

Figure 5. MGRO J2031+41: distributions of the parameter used to estimate the photon spectrum, $\mathcal{F}$. The spectrum fit is performed in the $\mathcal{F}$ space. Black squares represent the data. Red triangles and blue circles represent the simulated distributions for the best fit assuming a power law with a cutoff and a simple power law, respectively. The unit on the $y$-axis is the weighted excess per day, the unit on the $x$-axis is the $\mathcal{F}$ value. The error bars are the statistical uncertainties. (A color version of this figure is available in the online journal.)

other hand, the simple power-law model $\left(\alpha=2.78_{-0.10}^{+0.10}\right)$, already disfavored by the $F$-test, does not agree with the ARGOYBJ results. As mentioned in the Introduction, the VERITAS count map of the region shows an excess associated with MGRO J2019+37 and a separate source in the vicinity, VER J2016+372, for which VERITAS reported a flux of $1 \%$ of the Crab Nebula flux above $1 \mathrm{TeV}$ and a hard spectrum with a photon index of $2.1_{-0.4}^{+0.4}$ (Aliu et al. 2011). The hard spectrum VERITAS reports for VER J2016+372 agrees well with the hard spectrum measured by Milagro for MGRO J2019+37 in the case of the power law plus exponential cutoff assumption. However, if VER $\mathrm{J} 2016+372$ contributes to the flux of MGRO J2019+37, then it is a very small amount, since the flux of MGRO J2019+37 is about $80 \%$ of the Crab Nebula and VER J2016+372 is separated from the larger VERITAS excess associated with MGRO J2019+37. VERITAS has not reported a spectrum and flux for the latter excess, yet.

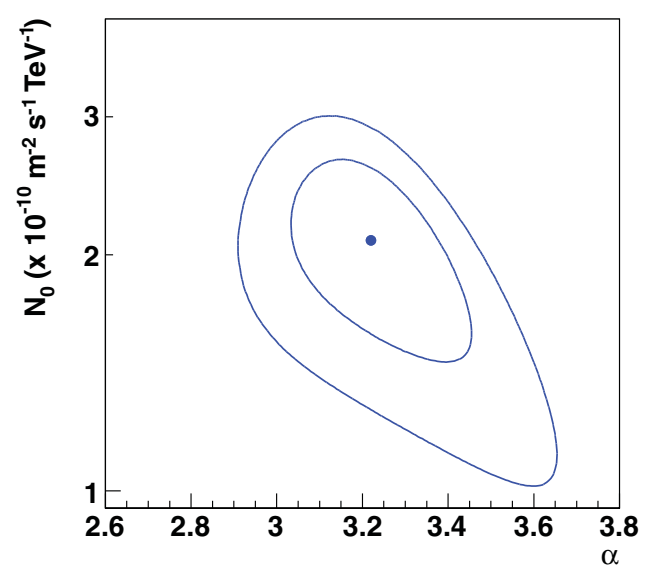

Figure 6. MGRO J2031+41: $1 \sigma$ and $2 \sigma$ CL contours for the power-law fit. The point indicates the best-fit result (minimum $\chi^{2}$ ).

(A color version of this figure is available in the online journal.)

\subsection{MGRO J2031+41 Spectrum}

The $\mathcal{F}$ distribution from data and those simulated for the two best-fit models are shown in Figure 5. MGRO J2031+41 accounts for $\sim 6 \%$ of the excess from the entire Cygnus region.

Figures 6 and 7 show the $1 \sigma$ and $2 \sigma$ contours from the $\chi^{2}$ fit for the two tested hypotheses.

Best-fit spectral parameters are summarized in Table 3.

For the power-law hypothesis, the best-fit parameters are $N_{10 \mathrm{TeV}}=2.1_{-0.6}^{+0.6} \times 10^{-10} \mathrm{~s}^{-1} \mathrm{~m}^{-2} \mathrm{TeV}^{-1}$, and $\alpha=3.22_{-0.18}^{+0.23}$. The $\chi^{2}$ is 5.06 ( $7 \mathrm{dof}$ ), with a $\chi^{2}$ fit probability of 0.65 .

For the power law with cutoff hypothesis, the best-fit parameters are $N_{10 \mathrm{TeV}}=5_{-3}^{+157} \times 10^{-10} \mathrm{~s}^{-1} \mathrm{~m}^{-2} \mathrm{TeV}^{-1}, \alpha=2.7_{-3.3}^{+0.7}$, and $E_{c}=21_{-18}^{+\infty} \mathrm{TeV}$. In this case, we are not able to constrain the upper limit of the $1 \sigma$ uncertainty for the cutoff energy (see the right panel of Figure 7). The maximum $E_{c}$ simulated value is $1000 \mathrm{TeV}$, an order of magnitude above the highest detected energy. The $\chi^{2}$ is 3.35 ( 6 dof), with a $\chi^{2}$ fit probability of 0.76 . The best-fit spectra for the two models are shown in Figure 8.

The improvement in the fit obtained with the more complex model is not significant, according to the $F$-test, and none of the parameters in the cutoff model are well constrained. Therefore, 

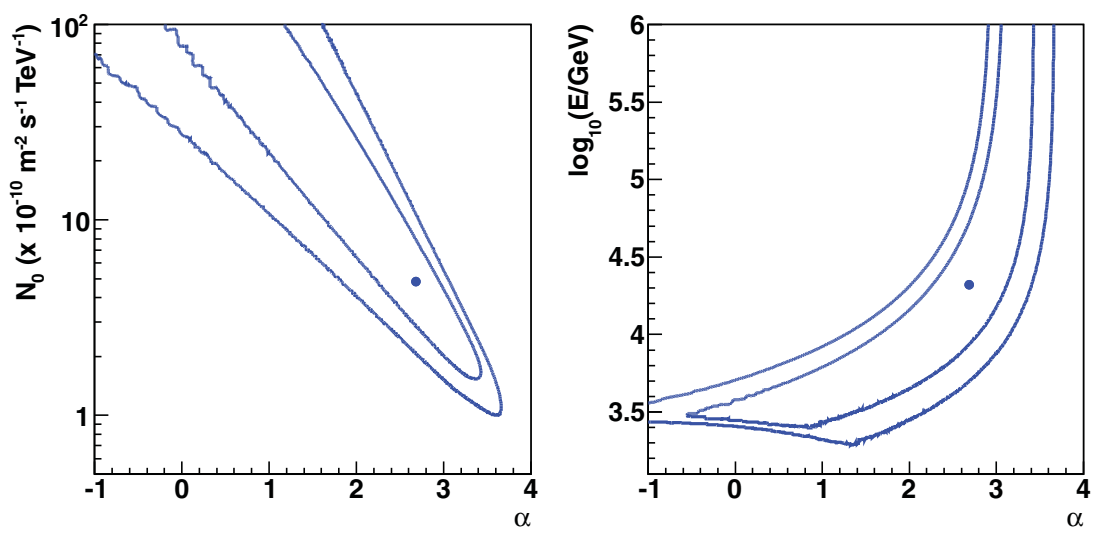

Figure 7. MGRO J2031+41: $1 \sigma$ and $2 \sigma$ CL contours for the power law with cutoff fit. Left panel: $N_{10} \mathrm{TeV}$ vs. $\alpha$ projection. Right panel: $E_{c}$ vs. $\alpha$ projection. The points indicate the best-fit result (minimum $\chi^{2}$ ). The right panel shows that the present analysis is unable to constrain the upper limit of the energy cutoff within $1 \sigma$.

(A color version of this figure is available in the online journal.)

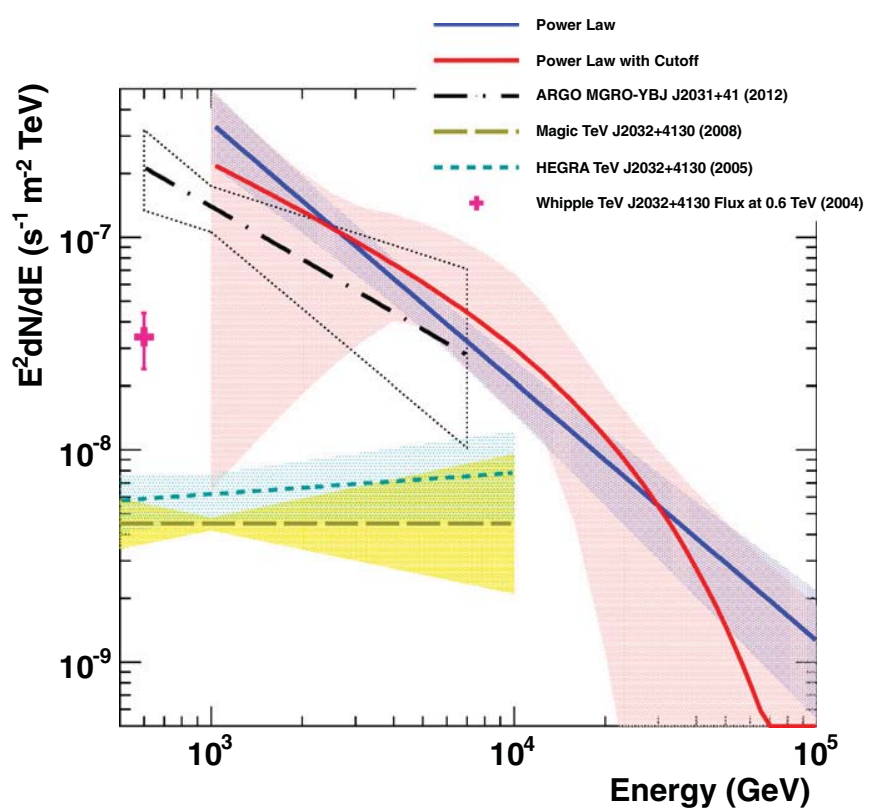

Figure 8. MGRO J2031+41: energy spectra. The power-law model is shown in blue and the power law with cutoff model is shown in red. These two hypotheses give a similar $\chi^{2}$ fit probability. The pink cross is the Whipple flux at $0.6 \mathrm{TeV}$ (Lang et al. 2004). The fine-dashed cyan line and the dashed yellow line are the HEGRA and MAGIC best fits (Aharonian et al. 2005; Albert et al. 2008), respectively. The dot-dashed black line is the ARGO-YBJ best fit (Bartoli et al. 2012). The shadowed area represents the $1 \sigma$ band.

(A color version of this figure is available in the online journal.)

the simple power law is to be preferred over the power law with the exponential cutoff.

The flux of MGRO J2031+41 at 20 and $35 \mathrm{TeV}$, measured by previous Milagro analyses (Abdo et al. 2007b, 2009b), is in agreement with the results presented here. Results from ARGO-YBJ, MAGIC, HEGRA, and Whipple (Bartoli et al. 2012; Albert et al. 2008; Aharonian et al. 2005; Lang et al. 2004) are shown in Figure 8. The HEGRA and MAGIC models are mutually consistent, but they disagree with our best fit in terms of integral flux in the overlapping energy range. Between 1 and $10 \mathrm{TeV}$, the flux measured by MAGIC accounts for only $\sim 3 \%$ of the flux measured by Milagro. The spectrum as measured by the air Cherenkov telescopes (ACTs) is also much harder $(\alpha \sim 2)$ than the Milagro power-law best fit $(\alpha \sim 3)$. This discrepancy could be explained by the following two facts.
First, the angular resolution of HEGRA and MAGIC $(<0.1)$ is much better than that of Milagro (0.3-0.7). The same is true for ARGO-YBJ, which, with an angular resolution between 0.47 and 2.8 , measured an emission consistent with the results presented here. The Whipple flux was measured at $0.6 \mathrm{TeV}$, with a PSF of 0.21, and lies between the ARGO-YBJ measurement and the HEGRA and MAGIC measurements. The measurement presented in this paper would therefore include photons coming from a larger region around the nominal source position compared to ACTs. Second, the way the background is computed is also different. Whipple used the ON/OFF method and observed the source with a significance of $3.3 \sigma$, while MAGIC primarily was operated in wobble mode $(5.6 \sigma)$. A possible explanation of the presented results is that $\mathrm{TeV} \mathrm{J} 2032+4130$, whose extension is slightly larger than the HEGRA and MAGIC angular resolution, is surrounded by an extended emission, $\sim 4^{\circ} \times 3^{\circ}$ according to the Milagro map (see Figure 9 discussed in Section 3.3), and according to the previously reported diameter of MGRO J2031+41 of 3.0 that was derived using a two-dimensional Gaussian fit (Abdo et al. 2007b; see also Table 1). Therefore, we believe that Milagro and ARGO-YBJ are not able to disentangle the extended emission from the central source and observe a higher flux. MAGIC and HEGRA, on the other hand, using ON/OFF and wobble mode might count the extended emission as background. As a result, the ACTs measure a fainter emission and a harder spectrum for TeV J2032+4130.

\subsection{Morphology}

The significance map of the Cygnus region for the last three years of Milagro data (2005-2008) is shown in Figure 9. An energy-dependent PSF smoothing is applied. The top panel shows the entire region in Galactic coordinates. Milagro positions for MGRO J2019+37 and MGRO J2031+41 are shown, as well as the high-significance Fermi LAT sources $(>10 \sigma)$ from the BSL catalog as reported in the second Fermi catalog (Nolan et al. 2012) and the extended Fermi Cocoon (Ackermann et al. 2011).

The Fermi counts map for photon energies above $10 \mathrm{GeV}$ overlaid with the Milagro significance contours in the Cygnus region is shown in Figure 10. Milagro detects an excess consistent with the position of the two pulsars PSR J2021+3651 and PSR J2032+4127, associated with MGRO J2019+37 and $\mathrm{J} 2031+41$, respectively, but no detection of significant emission coincident with PSR J2021+4026. 


\section{Cygnus Region}

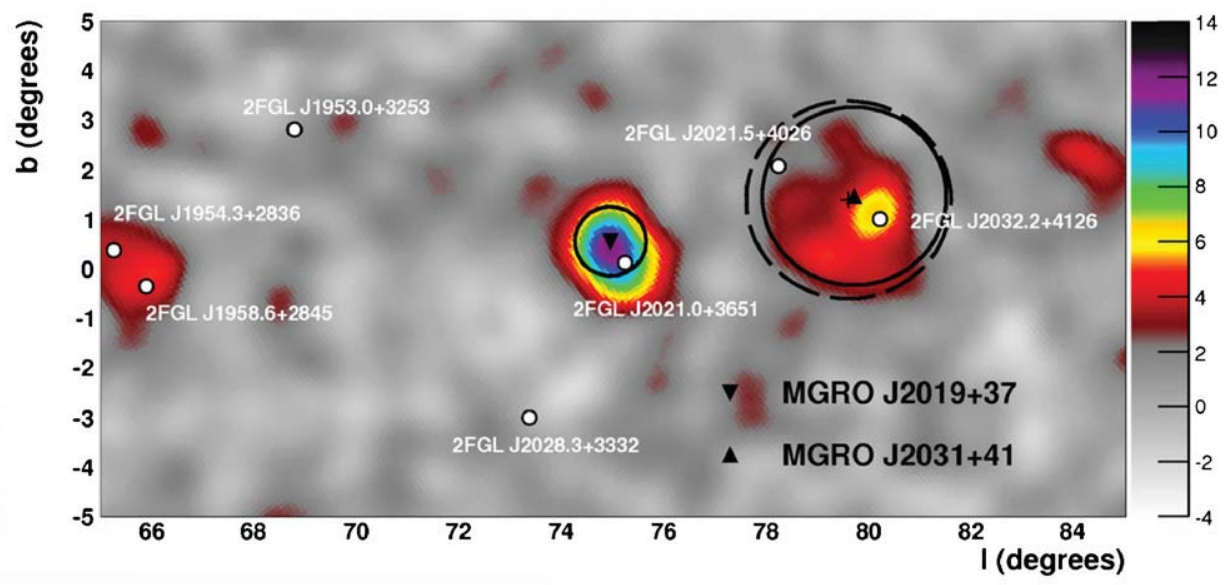

MGRO J2019+37

MGRO J2031+41
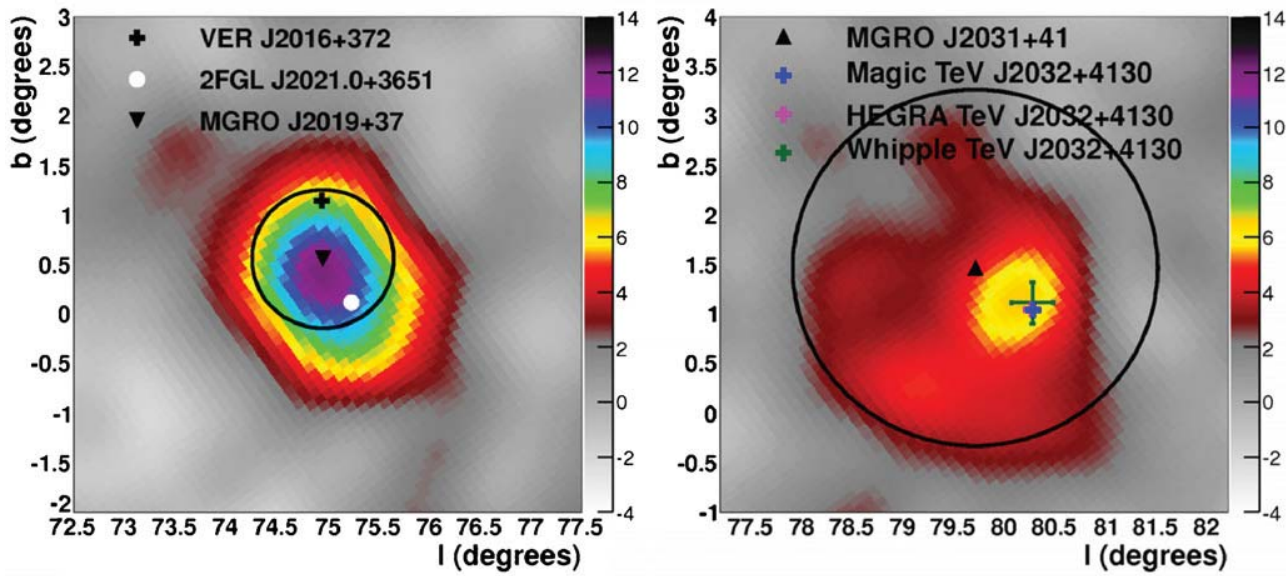

Figure 9. PSF-smoothed significance maps of the Cygnus region. Top panel: black triangles mark the positions of the two Milagro sources with the $1 \sigma$ radial width (solid circles), white-filled circles mark the Fermi BSL source positions as reported in the second Fermi catalog (the $1 \sigma$ error is smaller than the marker size). The Fermi Cocoon position is marked by the cross and the dashed circle is its $1 \sigma$ width. Bottom left panel: zoomed MGRO J2019+37 significance map. The circle represents the $1 \sigma$ width. The positions of VER J2016+372 and 2FGL J2021.0+3651 are also shown (see the text for a more detailed discussion of this region). Bottom right panel: zoomed MGRO J2031+41 significance map. The Milagro position with the $1 \sigma$ width is shown in black. The crosses mark the Whipple (green), HEGRA (magenta), and MAGIC (blue) positions with their uncertainty.

(A color version of this figure is available in the online journal.)

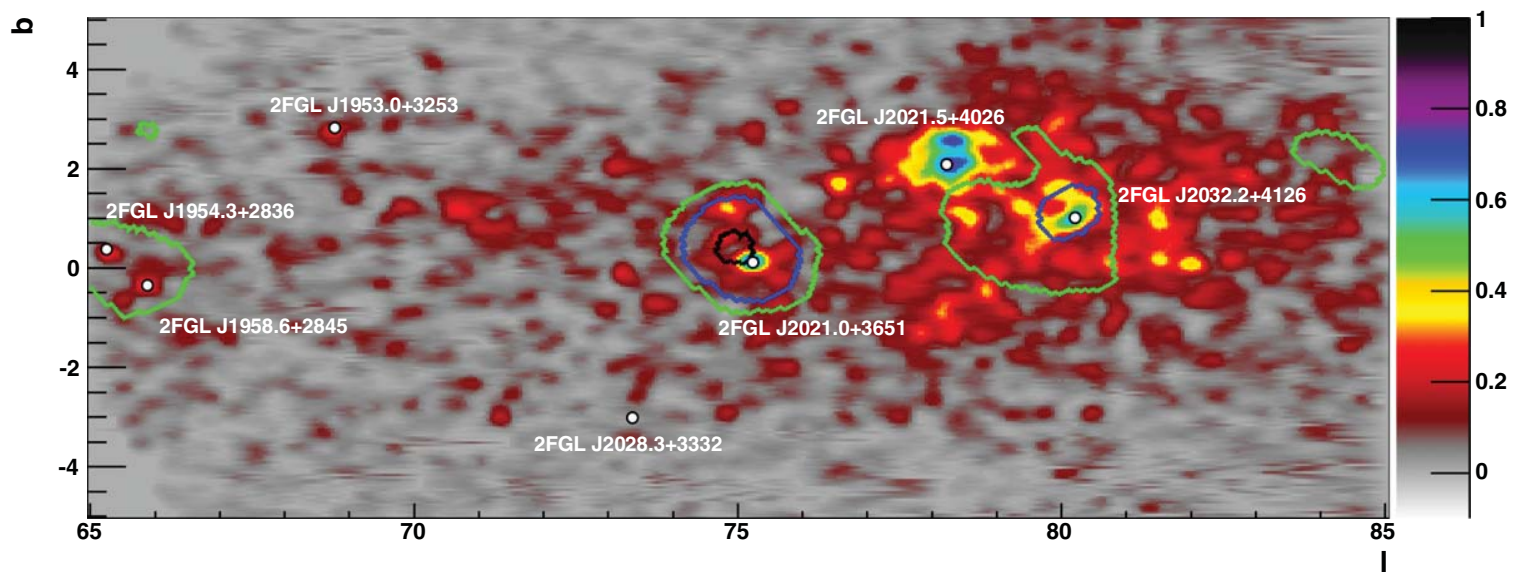

Figure 10. Fermi LAT counts maps of the Cygnus region above $10 \mathrm{GeV}$. We used Pass 7 photon data from 2008 August 4 15:43:37 to 2011 December 6 10:10:13 GMT LAT Data Server, zenith angle $<107^{\circ}$, DATA_QUAL $=1$, LAT_CONFIG $=1$. A Gaussian smoothing is applied for display purposes. Circles mark the position of the Fermi BSL sources as reported in the second Fermi catalog. The green, blue, and black lines are the Milagro $3 \sigma, 5 \sigma$, and $11 \sigma$ level significance contours, respectively. (A color version of this figure is available in the online journal.)

MGRO J2019+37 is observed with a pre-trials significance of $12.4 \sigma$ in the complete Milagro data set collected over eight years of operation. Its extension is obtained with the two- dimensional Gaussian fit discussed in Section 3. The fit results in $\sigma=0.7$, slightly larger than the angular resolution of the detector in its final configuration. There is evidence from 
VERITAS that the extended source Milagro observes is the result of the superimposition of close point-like sources (Aliu et al. 2011).

MGRO J2031+41, observed with a pre-trials significance of $7.6 \sigma$ in the complete Milagro data set, is an extended source, with $\sigma=1.8$. It shows a central core with a higher significance ( $>6 \sigma$ pre-trials) surrounded by a broader region (approximately $3^{\circ} \times 4^{\circ}$ ) with a lower significance ( $>4 \sigma$ pre-trials). TeV $\mathrm{J} 2032+4130$ lies within the central core, and the Fermi Cocoon $1 \sigma$ circle $\left(\sigma=2^{\circ}\right)$ completely includes MGRO J2031+41. However, the Fermi analysis (Ackermann et al. 2011) shows that the Cocoon has an elongated shape, poorly correlating with the Milagro contours, and the possible association between the Cocoon and the extended Milagro excess remains unclear.

The spectral fit results do not change if the maximum significance position is chosen instead of the two-dimensional Gaussian fit position.

\section{CONCLUSIONS}

We present the spectra of the two brightest Milagro sources in the Cygnus region using a new analysis technique applied to the last three years of data collected by the Milagro experiment.

MGRO J2019+37 is observed with a pre-trials significance over $12 \sigma$ in the complete Milagro data set during eight years of operation. Its emission is well fitted by a power law with an exponential cutoff $\left(E_{c}=29_{-16}^{+50} \mathrm{TeV}\right)$ and a hard asymptotic spectral index $\left(\alpha=2.0_{-1.0}^{+0.5}\right)$. The simple power law is disfavored. An $F$-test (Bevington \& Robinson 2003; Bates \& Watts 2007) for the drop in $\chi^{2}$ when adding the cutoff parameter to the fit gives a probability of $6 \times 10^{-4}$ of having seen a larger change, if the simpler power law without exponential cutoff were the correct model. When excluding the highest (two highest) $\mathcal{F}$ bin(s), this value goes up to $4 \times 10^{-3}\left(6 \times 10^{-2}\right)$. The TeV excess measured by Milagro from MGRO J2019+37, spatially associated with the Fermi LAT pulsars J2018.0+3626 and $\mathrm{J} 2021.0+3651$, has been confirmed by VERITAS, and it is likely produced by several nearby unresolved sources. The flux of these sources has not been reported by VERITAS, yet, though a separate source in the vicinity, VER J2016+372, is measured to have $1 \%$ of the Crab Nebula above $1 \mathrm{TeV}$ and a spectrum with a photon index of $2.1_{-0.4}^{+0.4}$ (Aliu et al. 2011). ARGO-YBJ does not detect a significant emission from MGRO J2019+37, but the $90 \%$ CL upper limits do not conflict with the Milagro best-fitting model.

The emission from MGRO J2031+41 (7.6 $\sigma$ pre-trials significance in the complete Milagro data set) is modeled by a power law with $\alpha=3.22_{-0.18}^{+0.23}$. Our result, in particular the integral flux in the overlapping energy range, is consistent with previous measurements by ARGO-YBJ, but it disagrees with Whipple,
HEGRA, and MAGIC results for TeV J2032+4130, most likely because of the different PSF of the instruments and different background subtraction methods. MGRO J2031+41 appears to show a structured morphology, produced by the superimposition of a central point-like source, coincident with TeV J2032+4130, and an extended emission that contributes to the overall spectrum Milagro measures for MGRO J2031+41. This extended emission is possibly produced by either unresolved sources or interactions of cosmic rays with the local interstellar medium. The correlation between the $\mathrm{TeV}$ extended emission and the overlapping Fermi Cocoon is unclear and needs further studies.

HAWC, the next generation water Cherenkov observatory, will be able to produce a more accurate analysis of the $\mathrm{TeV}$ emission from the Cygnus region, with its improved sensitivity (10-15 times better than Milagro) and angular resolution.

We gratefully acknowledge Scott Delay and Michael Schneider for their dedicated efforts in the construction and maintenance of the Milagro experiment. This work has been supported by the National Science Foundation (under grants PHY-0245234, -0302000, -0400424, -0504201, -0601080, -1002445, and ATM-0002744), the US Department of Energy (Office of High-Energy Physics and Office of Nuclear Physics), the Los Alamos National Laboratory, the University of California, and the Institute of Geophysics and Planetary Physics.

\section{REFERENCES}

Abdo, A. A., Allen, B., Aune, T., et al. 2008, ApJ, 688, 1078 Abdo, A. A., Allen, B., Berley, D., et al. 2007a, ApJ, 658, L33 Abdo, A. A., Allen, B., Berley, D., et al. 2007b, ApJ, 664, L91 Abdo, A. A., Ackermann, M., Ajello, M., et al. 2009a, ApJS, 183, 46 Abdo, A. A., Allen, B. T., Aune, T., et al. 2009b, ApJ, 700, L127 Abdo, A. A., Allen, B. T., Aune, T., et al. 2012, ApJ, 750, 63

Ackermann, M., Ajello, M., Allafort, A., et al. 2011, Science, 334, 1103 Aharonian, F., Akhperjanian, A., Beilicke, M., et al. 2005, A\&A, 431, 197 Albert, J., Aliu, E., Anderhub, H., et al. 2008, ApJ, 675, L25 Aliu, E. for the VERITAS Collaboration 2011, arXiv:1110.4656 Allen, B. 2007, PhD thesis, University of California, Irvine Atkins, R., Benbow, W., Berley, D., et al. 2003, ApJ, 595, 803 Bartoli, B., Bernardini, P., Bi, X. J., et al. 2012, ApJ, 745, L22 Bates, D. M., \& Watts, D. G. 2007, Nonlinear Regression Analysis and Its Applications (1st ed.; USA: Wiley-Interscience)

Bevington, R. P., \& Robinson, D. K. 2003, Data Reduction and Error Analysis for the Physical Sciences (3rd ed.; Boston, MA: McGraw-Hill)

Hunter, S. D., Bertsch, D. L., Catelli, J. R., et al. 1997, ApJ, 481, 205

Lang, M. J., Carter-Lewis, D. A., Fegan, D. J., et al. 2004, A\&A, 423, 415

LAT Data Server, http://fermi.gsfc.nasa.gov/ssc/data/access/

Moskalenko, I. V., \& Strong, A. W. 1998, ApJ, 493, 694

Moskalenko, I. V., \& Strong, A. W. 2000, Ap\&SS, 272, 247

Nolan, P. L., Abdo, A. A., Ackermann, M., et al. 2012, ApJS, 199, 31

Strong, A. W., \& Moskalenko, I. V. 1998, ApJ, 509, 212

The Fermi LAT Collaboration. 2012a, A\&A, 538, A71

The Fermi LAT Collaboration. 2012b, ApJS, 199, 31 